\title{
Erratum: Do the Walkability and Urban Leisure Amenities of Neighborhoods Affect the Body Mass Index of Individuals? Based on a Case Study in Seoul, South Korea
}

\author{
Yunwon Choi ${ }^{1}$ and Heeyeun Yoon ${ }^{2,3, *}$ \\ 1 Interdisciplinary Program in Landscape Architecture, Seoul National University, 1 Gwanak-ro, Gwanak-gu, \\ Seoul 151-742, Korea; yunwon.choi@snu.ac.kr \\ 2 Department of Landscape Architecture and Rural Systems Engineering, College of Agriculture and Life \\ Sciences, Seoul National University, 1 Gwanak-ro, Gwanak-gu, Seoul 151-921, Korea \\ 3 Research Institute of Agriculture and Life Sciences, Seoul National University, 1 Gwanak-ro, Gwanak-gu, \\ Seoul 151-921, Korea \\ * Correspondence: hyyoon@snu.ac.kr
}

Received: 14 July 2020; Accepted: 15 July 2020; Published: 17 July 2020

check for updates

The grant number in the acknowledgement was incorrect. The correction is provided below.

\section{Incorrect Grant Number}

This work was supported by the BK 21 Plus Project (Seoul National University Interdisciplinary Program in Landscape Architecture, Global leadership program toward innovative green infrastructure); the Korea Environmental Industry and Technology Institute (KEITI) (Grant number: 2014-001-310007); the Ministry of Education of the Republic of Korea and the National Research Foundation of Korea (Grant number: NRF-2017S1A5A8020226); the Creative-Pioneering Researchers Program through Seoul National University (Grant number: 500-20170162); the Ministry of Education of the Republic of Korea and the National Research Foundation of Korea (Grant number: NRF-2017S1A3A2066771); and the Korea Environmental Industry and Technology Institute (KEITI) through the Climate Change Response Technology Project, funded by the Korea Ministry of Environment (MOE) (201800131002).

\section{Correct Grant Number}

This work was supported by the Creative-Pioneering Researchers Program through Seoul National University (Grant number: 500-20170162), the Ministry of Education of the Republic of Korea and the National Research Foundation of Korea (Grant number: NRF-2017S1A3A2066771) and the Korea Environmental Industry and Technology Institute (KEITI) (Grant number: 2014-001-310007).

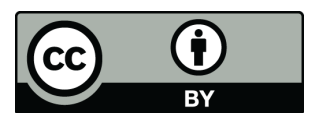

(C) 2020 by the authors. Licensee MDPI, Basel, Switzerland. This article is an open access article distributed under the terms and conditions of the Creative Commons Attribution (CC BY) license (http://creativecommons.org/licenses/by/4.0/). 